\title{
Antagonistic effects of chemical mixtures on the oxidative stress response are silenced by heat stress and reversed under dietary restriction
}

\author{
The oxidative stress response to chemical mixtures
}

\author{
Karthik Suresh Arulalan ${ }^{1, \bullet}$, Javier Huayta ${ }^{1, \bullet}$, Jonathan W. Stallrich ${ }^{2}$, and Adriana San-Miguel ${ }^{1, *}$ \\ ${ }^{1}$ Department of Chemical and Biomolecular Engineering, North Carolina State University, Raleigh, North \\ Carolina, USA. \\ ${ }^{2}$ Department of Statistics, North Carolina State University, Raleigh, North Carolina, USA. \\ *Corresponding author: asanmiguel@ncsu.edu \\ NC State University, Campus Box 7905, Raleigh, NC 27695-7905 \\ -These authors contributed equally to this work
}

Keywords: C. elegans, model organism, xenobiotics, oxidative stress, simplex centroid design, complex exposure

\begin{abstract}
Chemical agents released into the environment can induce oxidative stress in organisms, which is detrimental for health. Although environmental exposures typically incorporate multiple chemicals, organismal studies on oxidative stress derived from chemical agents commonly study exposures to individual compounds. In this work, we explore how chemical mixtures drive the oxidative stress response under various conditions in the nematode C. elegans, by quantitatively assessing levels of gst-4 expression. Our results indicate that naphthoquinone mixtures drive responses differently than individual components, and that altering environmental conditions, such as increased heat and reduced food availability, result in dramatically different oxidative stress responses mounted by $C$. elegans. When exposed to heat, the oxidative stress response is diminished. Notably, when exposed to limited food, the oxidative stress response specific to juglone is significantly heightened, while identified antagonistic interactions between some naphthoquinone components in mixtures are abolished. This implies that organismal responses to xenobiotics is confounded by environment and stressor interactions. Given the high number of variables under study, and their potential combinations, a simplex centroid design was used to capture such nontrivial response over the design space. This makes the case for the adoption of Design of Experiments approaches as they can greatly expand the experimental space probed in noisy biological readouts, and in combinatorial experiments. Our results also reveal gaps in our current knowledge of the organismal oxidative stress response, which can be addressed by employing sophisticated design of experiments approaches to identify significant interactions.
\end{abstract}

\section{Introduction}

Oxidative stress, which has deleterious effects on health ${ }^{1-3}$, can be induced by ROS (Reactive Oxygen Species) generated from oxidant chemicals. The effects of oxidant exposures on biological systems have been an important area of study, although these effects have been mostly analyzed by employing chemical exposures of individual components. ${ }^{4}$ However, realistic environmental exposures are mixtures of multiple components. $^{5-7}$ In addition, environmental factors, such as diet and temperature, can modulate the mechanisms by which chemicals induce toxicity and activate defense responses in living organisms. ${ }^{7,8}$ It is still unclear how chemical mixtures drive oxidative stress and how environmental conditions modify such 
responses. In this work, we analyze how mixtures of oxidant species, in particular naphthoquinones, differentially drive the oxidative stress response in the model organism C. elegans.

Naphthoquinones are strong oxidants. They are used as precursors of toxic industrial chemicals. ${ }^{9}$ They are also a product of fossil fuel combustion and atmospheric photochemical conversions, and are thus found in ambient particulate matter. ${ }^{10}$ Environmental exposures to naphthalene (a precursor for naphthoquinones) are significant as it is found in the atmosphere and in cigarette smoke. ${ }^{11-13}$ Naphthalene toxicity is thought to occur through the action of napthoquinones. ${ }^{14,15}$ Naphthoquinones have been hypothesized to induce oxidative stress through two distinct mechanisms ${ }^{16,17}$ : depletion of glutathione (an antioxidant that neutralizes ROS and counteracts xenobiotics by conjugation) through Michael reaction, or production of ROS through redox cycling. ${ }^{16}$ It is unclear if differences in the cytotoxic mechanisms amongst naphthoquinones could be reflected as differences in organismal responses to naphthoquinone mixtures. Naphtoquinone exposures at low concentrations can also induce beneficial effects. Simultaneous exposure to naphthoquinone derivatives such as juglone and plumbagin drive SKN-1/NRF-2 transcription factormediated hormesis in C. elegans at low concentrations, but turn toxic at higher concentrations. ${ }^{18}$ Naphthoquinones have also been shown to have anti-inflammatory effects in other model organisms. ${ }^{19}$ For example, allergen induced rats treated with juglone had a reduction in pulmonary eosinophils and bronchoalveolar lavage fluid. ${ }^{20}$ Given their prevalence as derivatives of naphthalene, and the differential responses to naphthoquinone exposures, it is critical to study the effects of naphthoquinone mixtures on organismal health.

The model system $C$. elegans facilitates studies on toxicity in a live organism with a well characterized nervous system, cell lineage, and physiology. C. elegans has proven to be a powerful model due to its small size, easy maintenance, and mapped genome and neuronal wiring. It enables in vivo studies using fluorescent markers due to their transparent bodies and ease of genetic manipulation. ${ }^{21}$ C. elegans has also been useful to study chemical mixture toxicity through growth and fertility assays. ${ }^{22-25} \mathrm{C}$. elegans and humans share concordant pathways, such as the insulin/IGF-1 signaling pathway (IIS), which regulates lifespan and healthspan extension driven by dietary restriction. ${ }^{26}$ Two-thirds of human proteins have homologs in C. elegans. ${ }^{26}$ C. elegans has been used to study mechanisms of toxicity identification, such as those produced by phorbol esters. ${ }^{26}$ Finally, toxicological studies have shown up to $69 \%$ concordance between $C$. elegans and mammalian toxicological data. ${ }^{27}$ Another study identified concordance between $C$. elegans data and that from rabbits and rats to be in the $45-53 \%$ range, just slightly lower than the concordance between rabbit and rat data $(58 \%){ }^{28}$

Defense mechanisms to metabolize and eliminate xenobiotics are evolutionarily conserved from single cell organisms to humans. ${ }^{29}$ In mammalian cells, NRF-1, NRF-2, NRF-3 are a class of NF-E2-related factor 2 (NRF) transcription regulators employed for such defense mechanisms. ${ }^{30}$ In C. elegans, the oxidative stress response pathway is activated to counteract the toxicity caused by oxidative stressors. ${ }^{31}$ The SKN-1 transcription factor, the functional ortholog of mammalian NRF-2, is major regulator of the oxidative stress response in C. elegans. ${ }^{30,32,33}$ It should be noted that SKN-1 diverges from NRF-2 in the way it binds to DNA. However, the similarities allow us to study SKN-1 in C. elegans as a model for mammalian NRF$2 .{ }^{30} \mathrm{SKN}-1$ has also been linked to other much broader homeostatic functions such as reducing stress, counteracting lipid accumulation, mitochondrial biogenesis and mitophagy, among others. ${ }^{30} \mathrm{SKN}-1$ activity in response to chemicals and heavy metals has been studied by examining expression of SKN-1-regulated genes using endogenously expressed fluorescent reporters. SKN-1 driven expression is mostly studied in the intestine, where digestion and detoxification occur. ${ }^{4,34-36}$ Multiple antioxidant response elements (AREs) containing genes are downstream targets of SKN-1, such as gst-4 and gcs-1, which encode for drugmetabolizing glutathione S-transferase (GST-4) and gamma-glutamyl cysteine synthetase (GCS-1), important in glutathione synthesis, respectively. For example, prior work by Crombie et al. focused on studying the effect of environmental factors on the oxidative stress response to juglone using a two level 
full factorial design, by monitoring gst-4 expression. ${ }^{37} \mathrm{gst}-4$ is commonly used as proxy for SKN-1 activity and thus activation of the oxidative stress response. ${ }^{38-40}$

In this study, we analyzed the effects of naphthoquinone mixtures on the $C$. elegans oxidative stress response under various environmental conditions, as determined by a gst-4 translational fluorescent reporter. We follow a Design of Experiments (DoE) approach that enables systematic examination of the input factors to determine the individual and combinatorial influence on the measured response, ${ }^{41,42}$ while avoiding the unfeasible number of experiments required for full factorial designs with multi-level factors. This approach minimizes the number of experimental runs necessary to measure interactions between three naphthoquinones and two environmental conditions, while enabling comparisons from independent biological populations. We quantify response surfaces for these ternary mixtures under different conditions. We find that naphthoquinone mixtures drive antagonistic interactions, but these interactions are drastically modified by dietary restriction. On the other hand, heat stress abolishes oxidative stress response to both individual components and mixtures of naphthoquinones. This gst-4 response is independent of the IIS factor DAF-16. The ROS levels and resistance to acute oxidative stress that result from exposure to naphthoquinone mixtures are also modulated by environmental factors, in the opposite direction as gst-4 expression.

\section{Materials and methods}

\section{Strain maintenance}

C. elegans was maintained on standard Nematode Growth Medium (NGM) plates seeded with OP50 E. coli bacteria and maintained at $25^{\circ} \mathrm{C}$. Worms at day 1 of adulthood were bleached and age synchronized using standard protocols, ${ }^{43}$ and grown to the young adult stage before application of oxidative stress. The strains used in the experiments were CL2166: $d v L s 19$ [pAF15 (gst-4P:GFP::NLS)], MAH97 muIs109 [daf16p::GFP::DAF-16 cDNA + odr-1p::RFP], and N2 (wild type) which were obtained from the Caenorhabditis Genetics Center. Animals were exposed to either control (S-Medium) or naphthoquinone mixtures in liquid culture in the presence of HB101 E. coli as a food source, based on established procedures. ${ }^{44} E$. coli was grown in LB media in the presence of $4 \mathrm{mM}$ streptomycin. Bacteria were washed thrice with SB media, pelletized, and resuspended in S-Medium at a concentration of $100 \mathrm{mg} \mathrm{mL}^{-1}$. Bacteria was then killed by heat treatment at $65{ }^{\circ} \mathrm{C}$ for a period of $45 \mathrm{mins},{ }^{37}$ to avoid bacterial metabolism to confound results.

\section{Chemical preparation}

Juglone, 1, 4-naphthoquinone, and plumbagin (Figure 1A) were sourced from Fisher Scientific and stored according to supplier guidelines. $100 \mathrm{mM}$ stock solutions were prepared for 1, 4-naphthoquinone and plumbagin by dissolving the powdered compounds in DMSO and stored at $-20{ }^{\circ} \mathrm{C}$. Juglone stock was prepared fresh before the start of each experiment, given its low stability. ${ }^{4}$

\section{Experimental design}

An experimental design was generated to study the effect of oxidant mixtures at ad libitum feeding ( $2 \mathrm{x}$ $10^{10}$ cells $\mathrm{mL}^{-1}$ ) and no heat stress conditions, using a simplex centroid design ( 7 points in the experimental space, Figure 1B). The simplex centroid design was chosen as it entails a low number of experimental points, thus facilitating performing more replicates. This design entailed 40 runs split into 10 groups of 4 runs (where each design point had $5-7$ replicates). To assign simplex centroid points to groups, we started from an I-optimal design generated in JMP Pro v 14.2 with 21 points in the experimental space, which already assigns each point to a group. These I-block points were then approximated to the closest point in the simplex centroid design, and their assignment to a group from the I-block design was maintained. An additional control run was tested along with each group to account for differences in populations due to any 
experimental variability (ambient temperature, humidity, etc.). A population of animals from a single NGM plate was thus divided into 5 runs (4 for design points, and one as a control).

To study the effects of process conditions on responses induced by chemical mixtures, a split plot mixture experiment was performed. The whole plot factors were the two environmental stressors and the split plot factors were the naphthoquinone mixtures. Two levels of heat exposures: $20^{\circ} \mathrm{C}$ and sublethal $33{ }^{\circ} \mathrm{C}$ were considered as the first environmental stressor. Two levels of HB101 bacterial diet regime - ad libitum $(2 \mathrm{x}$ $10^{10}$ cells $\left.\mathrm{mL}^{-1}\right)(\mathrm{AL})$ and dietary restriction $\left(2 \times 10^{9}\right.$ cells $\left.\mathrm{mL}^{-1}\right)(\mathrm{DR})$ was considered as the second environmental stressor. ${ }^{37,45}$ A total of 28 populations of animals were used for the split plot mixture experiments. Each population was split into five runs. One run, at process conditions $20^{\circ} \mathrm{C}$ and $\mathrm{ad}$ libitum diet named "overall control", was used to compare the 28 populations ( 7 points of simplex centroid design * 4 process conditions). Another run named "experimental control" was reserved for testing the effect of individual environmental stressor, i.e., a specific heat level and diet combination with no oxidant. Thus, each of the four process conditions were replicated 7 times across the 28 populations. The remaining three runs were exposed to the same combination of diet and heat exposure as the experimental control, but each was exposed to a different chemical mixture. These were assigned to groups similar to the previous experiment, using experimental points from a simplex centroid design. Hence, a total of 84 runs ( 7 points of the simplex centroid design $* 3$ replicates $* 4$ process conditions) and 56 control runs were used to study the effects of heat stress and dietary restriction on the naphthoquinone mixtures. The overall controls were plotted as Xbar and S control chart to ensure that populations were statistically comparable with each other (Figure S1).

\section{Application of oxidative stress and fluorescence imaging}

Approximately 30-40 age-synchronized worms were loaded into a well of a 24-well plate containing the oxidant and HB101 bacteria in S-Medium. Control experiments were conducted with an empty vector of $1 \% \mathrm{v} / \mathrm{v}$ DMSO. Animals were exposed to the oxidants for a period of 8 hours at a temperature of $20 \pm 1{ }^{\circ} \mathrm{C}$. For Pgst-4::GFP imaging, worms were immobilized using a drop of $4 \mathrm{mM}$ tetramisole on dried $2 \%$ agarose pads. Worms were imaged using a wide field inverted fluorescence microscope Leica DMi8 at 10x magnification after the exposure period. A Spectra X LED illumination system centered at $470 \mathrm{~nm}$ was used for excitation, and a Hamamatsu Orca Flash 4.0 16-bit digital CMOS camera was used for image acquisition. A dose response to individual compounds was first quantified by exposing animals in increasing concentrations from $0 \mu \mathrm{M}$ to $100 \mu \mathrm{M}$ for a period of 8 hours. The naphthoquinone mixtures were applied based on the designs described the previous section.

\section{Quantitative image processing}

GFP driven by the gst-4 promoter is observed throughout the animal, and its intensity was quantified to estimate oxidative stress response in vivo. Images were analyzed using a MATLAB script that generates a binary mask to identify a single worm per image. The MATLAB regionprops function was then used to quantify the mean intensity of the animal by overlapping the binary mask over the original image (Figure 1C).

\section{RNAi by feeding}

To measure the influence of $d a f-16$ on the $g s t-4$ response to environmental conditions, age-synchronized worms were grown from the egg stage to day 1 of adulthood at $20^{\circ} \mathrm{C}$ in NGM plates containing HB101 bacteria containing the dsRNA-producing vector from the Ahringer library (acquired from Source Biosciences). ${ }^{46,47}$ Animals were then bleached following standard protocols. ${ }^{43}$ The eggs obtained were deposited in new NGM plates containing HB101 bacteria carrying the daf-16 RNAi vector. Animals were grown to young adulthood at $20^{\circ} \mathrm{C}$, and then exposed to oxidative stress as previously described. 


\section{ROS detection}

To measure ROS levels, 30 animals, exposed to oxidants as described above, were washed twice in M9, and then stained for 2 hours in $1 \mathrm{~mL}$ of M9 containing $150 \mu \mathrm{M}$ 2',7'-dichlorofluorescein diacetate (DCFDA, Sigma) while rotating in the dark. Worms were then washed twice with M9, and transferred to $2 \%$ agarose pads on glass slides, covered, and immediately imaged within 30 minutes of washing out the DCFDA. ${ }^{48,49}$ Imaging was performed as previously described. Images were analyzed in ImageJ, where average intensity of the head region was scored.

\section{Survival assay and lifespan curves}

To measure survival of animals to acute oxidative stress, 50 worms, exposed to oxidants as described above, were washed twice in M9, and then transferred to a 24 -well plate containing $1 \mathrm{ml} \mathrm{M} 9$ with $250 \mu \mathrm{M}$ juglone. Animals observed as rigid and immobile after imparting movement to the liquid in the well were scored as dead. Animals were scored for survival every 30 minutes for the first 2 hours and every 1 hour thereafter until all animals were scored as dead. ${ }^{4,45}$ Lifespan curves and statistical analysis of mean lifespan were performed using the Online Application for Survival Analysis 2 (OASIS 2). ${ }^{50}$

\section{Statistical analysis}

Data was compiled and analyzed using JMP Pro v.14.2. The standard least squares second order Scheffe model accounting for the individual components, mixture interactions, and effect of the process variables was used..$^{51}$ The block effect in the first design and the split plot in the second design were taken to be random effects. All effects, including interactions between the mixtures and process variables, with $\mathrm{p}$ value smaller than 0.05 during statistical testing $(\alpha<0.05)$ were considered as significant. Since this approach means dealing with multiple $C$. elegans populations (a population is considered animals cultured on the same NGM plate), we monitored both the mean and variability of gene expression to ensure that population responses are within statistical limits of each other. To assess whether different populations were comparable, we used control limits. A population plotted within the control limits is equivalent to failing to reject the null hypothesis of statistical control (i.e., all populations have equal means), and a population plotted outside the control limits is equivalent to rejecting the null hypothesis. The process mean was monitored using the $\mathrm{X}$ bar chart, while process variability was monitored using the $\mathrm{S}$ chart. ${ }^{52}$ The responses of the controls were plotted using an Xbar (average) and S (standard deviation) control chart (Figure S1).

\section{Results}

\section{Dose dependency of $g s t-4$ response}

230

231

232

233

234

235

236

237

238

239

240

To identify relevant concentrations of chemical mixtures of plumbagin, 1, 4-naphthoquinone, and juglone, (Figure 1A, B, C) we first determined the dose-dependent $g s t-4$ activity to individual components (Figure 1D). Animals were exposed for 8 hours, based on prior studies that suggest this time is sufficient to observe a gst-4 response.$^{53,54}$ gst-4 belongs to a class of enzymes used to catalyze conjugation of glutathione with xenobiotics. Prior work determined that $g s t-4$ expression is increased in animals stressed with xenobiotics, while external ROS generated by hypoxanthine/XOD system, UV light, and heat did not elicit a response. ${ }^{40}$ As expected, we identified that as the naphthoquinones concentration increases, $g s t-4$ activity also increases and eventually saturates (Figure 1D), as described in previous studies. ${ }^{53,54}$ To assess the effect of mixture proportions on oxidative stress response, the total naphthoquinone dosage should remain constant. Based on these results, we fixed a combined total dosage of $30 \mu \mathrm{M}$ for mixture experiments, which allows studying the interactions of components at different proportions without potential saturation of the $g s t-4$ response. 
Sublethal doses in the range of $20-30 \mu \mathrm{M}$ for the 3 compounds under study are known to drive SKN-1dependent expression of $g s t-4$, and also induce a hormetic effect driven by SKN-1., ${ }^{4,3,37}$

\section{Naphthoquinone mixtures show antagonistic effects under ad libitum feeding and physiological} temperature conditions.

The first mixture experiments were performed under ad libitum feeding and no heat stress conditions based on the simplex centroid design. The average and standard deviations of the baseline gst-4 responses of controls run for each of the ten sets of experiments were compared to determine if runs were comparable to each other. All measurements are within statistical control (Figure S1A). We used a Scheffe model fit to develop a response surface (Figure 2A). The block effect added in the model indicates that populations 4, 6, and 10 exhibited a significant effect (Table S1), suggesting some experimental variation for these populations. However, these deviations caused by the block effects are accounted for in the model. The response surface indicates that the individual naphthoquinones induce a higher $g s t$ - 4 response than binary and ternary mixtures, suggesting an antagonistic interaction (Figure 2A, Table S1). Since animals were maintained at $25^{\circ} \mathrm{C}$ and shifted to $20^{\circ} \mathrm{C}$ for chemical exposure, a control exposure experiment at $25^{\circ} \mathrm{C}$ was tested to determine if the temperature shift could play a role in the observed responses. The control experiment maintained at $25^{\circ} \mathrm{C}$ shows the same $g s t-4$ expression trend as those exhibited by animals shifted to $20^{\circ} \mathrm{C}$ for exposure (Figure $\mathbf{S 2}$ and Figure $\mathbf{2 A}$ ): naphthoquinone mixtures are antagonistic in driving the gst-4 response.

Naphthoquinone mixtures induce different $g s t-4$ responses under different environmental conditions

Temperature and dietary intake have been shown to affect the oxidative stress response in C. elegans. ${ }^{33,37,55}$ Thus, we tested how exposure to heat stress and dietary restriction modified the gst-4 response to naphthoquinone mixtures by fitting the $g s t-4$ expression data to a mixed effects model. The overall control was plotted as Xbar and S chart and indicates that the populations are within statistical limits of each other (Figure S1B). Block effects analysis revealed population 5 exhibited a statistically significant effect compared to the other 27 populations (Table S2). However, the model corrects for the effect caused by the treatment application process on population 5 .

Heat stress and dietary restriction (DR) significantly affect the oxidative stress response, inducing a lower baseline gst-4 response than a control of $20^{\circ} \mathrm{C}$ and ad libitum conditions (Figure 2B). However, heat and dietary restriction induce differential responses in $g s t-4$ in the presence of naphthoquinone mixtures (Figure 3A, B). The ad libitum and $20^{\circ} \mathrm{C}$ response surface (Figure 3B) is the repetition of the first mixture experiment and was confirmed to not be statistically different (Figure 2A). The experimentally acquired data is represented as conventional bar plots in Figure S3. As the temperature is increased to $33{ }^{\circ} \mathrm{C}$, heat stress inhibits the $g s t-4$ response to pure components and mixtures, consistent with prior results. ${ }^{37}$ Dietary restriction reduces the baseline $g s t-4$ level (Figure 2B).$^{56}$ In contrast, reduced food concentration did not affect the $g s t-4$ response to 1,4-naphthoquinone and plumbagin (Figure 3B) and it drastically increased the response to juglone, as compared to ad libitum conditions. Furthermore, exposure to binary or ternary mixtures under dietary restriction did not result in antagonistic interactions observed in mixtures at $a d$ libitum dietary regime (Figure 3B, C). These results suggest that dietary restriction can differentially modulate the oxidative stress response to individual compounds, and significantly modify interactions amongst naphthoquinones.

We next asked whether these environmental modifications of the oxidative stress response to naphthoquinone mixtures could stem from differences in ROS levels, and if the gst-4 response exhibiting differential responses to mixtures and environmental variables would imply differences in organismal resistance to acute oxidative stress. These questions were addressed by measuring ROS levels through DCFDA staining of N2 animals (Figure 3D, E) and by assessing the survival of worms to acute oxidant 
exposures after exposure to low-level naphthoquinone mixtures (Figure 3F, S4). These experiments show that the presence of ROS in the worms is highest under conditions of dietary restriction at $33{ }^{\circ} \mathrm{C}$, which are also conditions with the lowest level of $g s t-4$ (Figure 3C). The differences in ROS levels between the populations exposed to heat stress could be explained by an increase formation of ROS in animals under glucose restriction, ${ }^{57}$ while high levels of glucose renders C. elegans more resilient to oxidative stress. ${ }^{58} \mathrm{In}$ this case, a parallel can be drawn between glucose and food availability. Animals exposed to acute juglone concentrations exhibit the lowest survival under heat stress (Figure 3F), which matches with the lowest observed levels of gst-4 (Figure 3C). These results suggest that environmental conditions that modulate the $g s t-4$ responses to naphthoquinone mixtures similarly affect the animal's resistance to oxidants, and that ROS levels are also increased in conditions that result in minimal gst-4 expression and highest susceptibility to acute juglone exposures.

Since dietary restriction modifies the gst-4 responses to naphthoquinones (individually and in mixtures), we then asked whether this effect could be modulated by the insulin/insulin-like signaling (IIS) pathway that can be activated with specific dietary restriction regimes. ${ }^{59}$ To address this question, we tested a possible dependence of the gst-4 response on $d a f-16$, the main regulator of the IIS pathway (Figure 3G, S5) ${ }^{60,61}$ Comparing the $g s t-4$ responses in the presence (Figure 3C) and absence (Figure 3G) of DAF-16 shows that the DR-dependent induction of $g s t-4$ under naphthoquinone exposures is independent of DAF16, suggesting an alternative pathway is at play. Likely skn-l itself regulates this interaction, since it is known to play a role in DR-induced lifespan extension. ${ }^{62}$ We also measured daf-16 responses to naphthoquinones mixture exposure by assessing the levels of a DAF-16::GFP fusion protein within intestinal cell nuclei. Like gst-4, daf-16 responses are inhibited by heat stress (Figure 3H). On the other hand, ternary naphthoquinone mixtures induced strong daf-16 responses in both DR and AL conditions. As mentioned above, under AL conditions, gst-4 exhibits a reduced signal for ternary mixtures (i.e., antagonistic interactions), which is not observed in daf-16 activity. This result suggests naphthoquinone mixtures induce strong daf-16 activity, which is inhibited by heat stress, and the daf-16 response to oxidants is not modulated by DR, potentially indicating that oxidative stress is prioritized over food intake for downstream signaling.

\section{Discussion}

In this work, we took advantage of a DoE approach to study the oxidative stress response driven by combinatorial exposures to naphthoquinones under a variety of environmental conditions. Unlike traditional full-factorial designs, which would entail an unfeasible number of experiments, using block effects and a simplex centroid design enabled performing experiments in a non-simultaneous and feasible manner. Initially, using mixed second order Scheffe model statistical analysis, we built response curve to ternary naphthoquinone mixtures under controlled environmental factors. The observed antagonistic interactions in Figure 2A could stem from different mechanisms of toxicity elicited by the mixture components. For instance, there are differences in the chemical reactivity of juglone and plumbagin. ${ }^{63}$ Naphthoquinones have been shown to result in toxicity through ROS generation or glutathione depletion. ${ }^{16,18}$ Juglone has relatively higher chemical activity and could undergo Michael's addition to glutathione even at lower doses. ${ }^{63,64} 30 \mu \mathrm{M}$ of 1, 4-naphthoquinone and plumbagin could cause oxidative stress through redox cycling at lower doses, and only at higher doses cause Michael's addition to glutathione. ${ }^{63,65}$ Mixtures of these chemicals could thus result in lower gst-4 activation than individual mixtures, if $g s t-4$ induction is more sensitive towards one of these toxicity mechanisms.

We further analyze if the response to naphthoquinones would be modified under different environmental conditions: food availability and temperature, and found drastic changes to response curves. The reduction in gst-4 expression levels by heat stress observed in Figure $\mathbf{2 B}$ could be explained by organismal prioritization of the heat shock response over the oxidative stress response, as previously suggested by 
of $c c t-4$ under dietary restriction, which encodes a chaperonin directly involved in SKN-1-dependent transcription of $g s t-4 .{ }^{56} \mathrm{We}$ also built response curves to ternary naphthoquinone mixtures under the four environmental conditions, which revealed significant interactions between chemicals. Surprisingly, these interactions were drastically modulated by environmental conditions of temperature and food availability. The identified gst-4 responses to naphthoquinones is abolished by heat stress, which could be attributed to prioritization of proteostasis over detoxification, where the HSF-1 driven heat stress response genes are upregulated to prevent protein misfolding. ${ }^{37}$

Dietary restriction also modulated stress response. Under ad libitum condition, individual components drove $g s t-4$ expression to similar levels, however, dietary restriction-induced oxidative stress response show high specificity for juglone, suggesting differences in organismal processing of similar oxidants. Although there is cross-regulation between the diet regulated DAF-16 and oxidative stress regulated SKN-1 pathways, ${ }^{66,67}$ RNAi experiments revealed that $g s t-4$ responses to mixtures are daf-16-independent. However, SKN-1 is also known to modulate DR-induced modulation of longevity, ${ }^{62}$ and is thus likely integrating signals for DR and oxidative stress and driving the observed interactions. Interestingly, daf-16 is activated by naphthoquinone mixtures in the absence of heat stress, recapitulating the $g s t-4$ inhibition by heat stress. In contrast, DR did not elicit higher daf-16 activation than AL conditions, suggesting organismal responses to oxidants are prioritized over reduced caloric intake. It is still unclear why the DR effects are specific to the oxidant type. Potentially, these differences could stem from differences in toxicity and detection mechanisms through chemosensation, as explained before, coupled with multiple transcriptional pathways interacting at the organismal level.

These findings highlight the importance of experimental analysis in realistic settings, where a variety of chemical components is present, and where environmental conditions vary significantly. In addition, the identified interactions between naphthoquinone mixtures, heat stress, and dietary restriction, shed light on organismal integration and processing of stressors and environmental factors. This could stem from differences in oxidant detection in C. elegans. For instance, low levels of $\mathrm{H}_{2} \mathrm{O}_{2}$ activate the $\mathrm{I} 2$ neuron, while paraquat only elicits a response at a very high concentration. ${ }^{68}$ The difference in the gst-4 response to individual compounds and mixtures could stem from the combined effects of differences in xenobiotic detection and the mechanisms of toxicity by the different naphthoquinones. This result indicates that individual components do not act through a singular mechanism, and that mixtures can drive significantly different organismal responses than individual components, even for highly similar chemical species. A detailed investigation on neuronal SKN-1 could help elucidate whether the interaction mechanisms between chemical mixtures and environments involve neuronal detection.

C. elegans are complex in nature and many factors could affect the response to oxidative stress, such as variation in developmental period, food availability on plate, temperature, as well as biological stochasticity. It is necessary to control and account for such effects as combinatorial mixture experiments cannot be performed using a single population. Control charts have proven to be useful in identifying potential problematic populations, which might show a different response. Such populations can have different biological activity and can affect the results of the experiments being performed. Adding block factors and split plots as random effects to the model also help us compare between populations, even if these exhibit differences that could come from experimental or biological variation. Thus, using a combination of mixture experiments and control charts we have highlighted the differential $g s t-4$ response by $C$. elegans to oxidative stressor mixtures under different environmental conditions. These results warrant further investigation of the different transcriptional pathways involved and shed light on how organisms respond to variable environments and realistic chemical exposures. 
A<smiles>O=C1C=C(Cl)C(=O)c2c(O)cccc21</smiles>

Plumbagin 1,4-Naphthoquinone

B

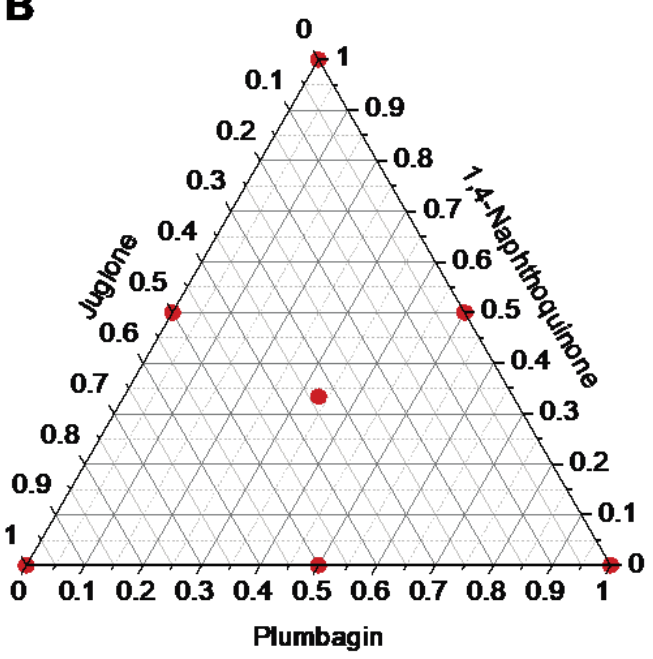

C

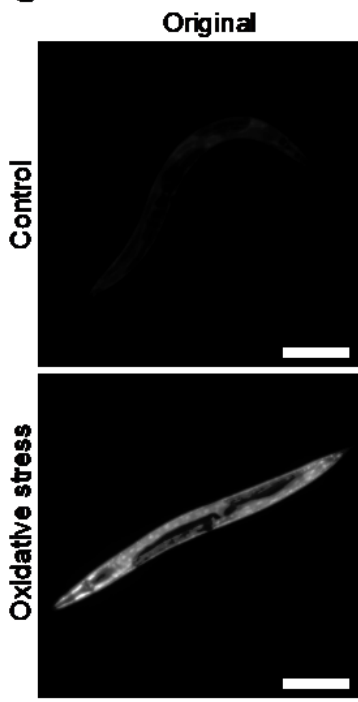

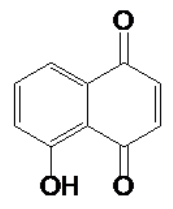

Juglone<smiles>O=C1C=CC(=O)c2ccccc21</smiles>

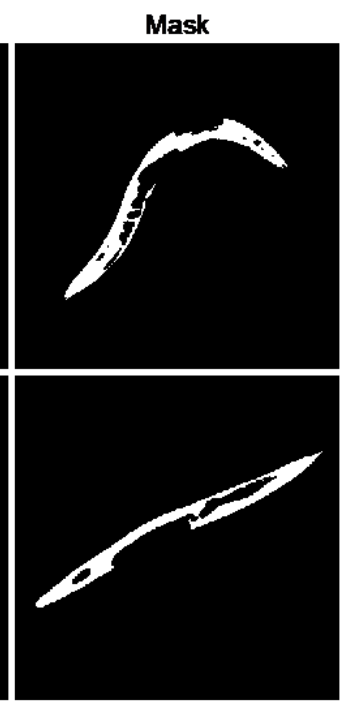

D
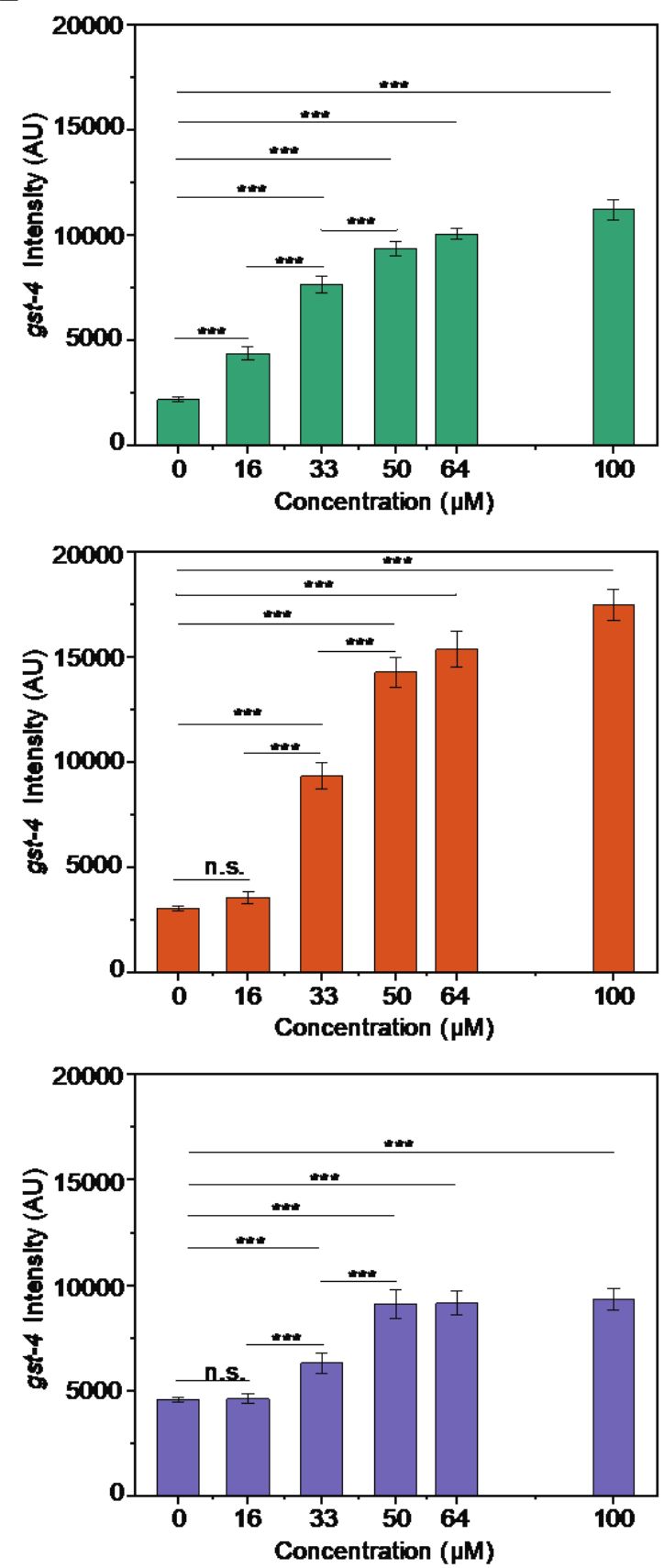

Figure 1: gst-4 response is dose dependent. A) Compounds used for oxidative assays. B) Simplex centroid design. C) CL2166 worms under control and oxidative stress with corresponding masks for extracting gst4 expression levels. D) gst-4 dose-dependent response to Plumbagin, 1, 4-Naphthoquinone, and Juglone (top to bottom). p $>0.05$ (n.s.), $\mathrm{p}<0.001$ (***). Error bars are SEM. All p-values were calculated using Tukey HSD for all pairwise comparisons after one-way ANOVA (Unequal Variances) comparison in JMP 14.2. Scale bars are $200 \mu \mathrm{m}$. 
A
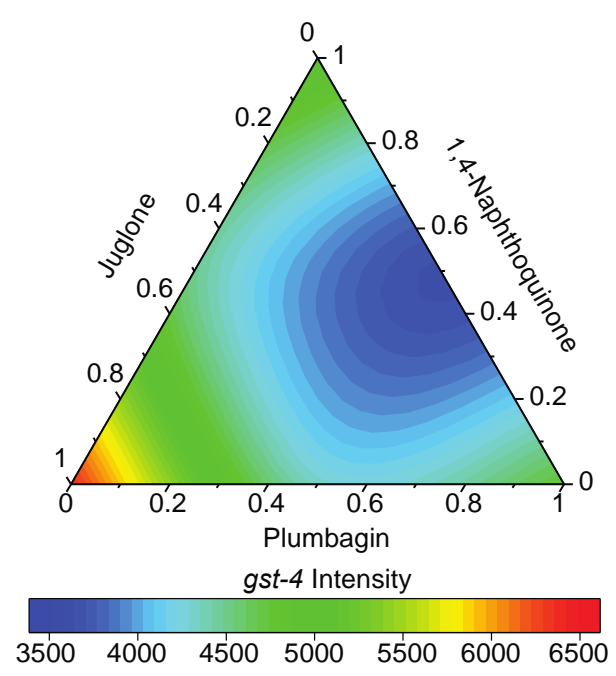

B

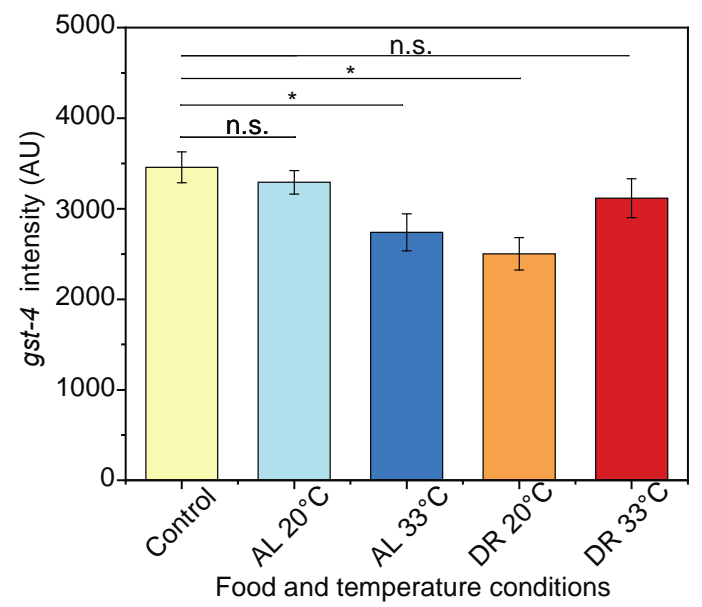

Figure 2: Naphthoquinone mixtures show no synergistic effects under ad libitum feeding and no heat stress conditions. A) Response surface of $g s t-4$ expression levels in CL2166 animals under oxidative stress, $20^{\circ} \mathrm{C}$, and ad libitum feeding. Response surface modeled using standard least squares second order Scheffe model where main effects and interactions were tested for significance (Table S1). B) Testing for main effect of temperature and food concentration. $\mathrm{p}>0.05$ (n.s.), $\mathrm{p}<0.05(*)$. p-values were calculated using Dunnett's test with ad libitum, overall control as control after two-way ANOVA comparison in JMP 14.2. Error bars are SEM. A control at $25^{\circ} \mathrm{C}$ is represented as a bar plot in Figure S2. 
A

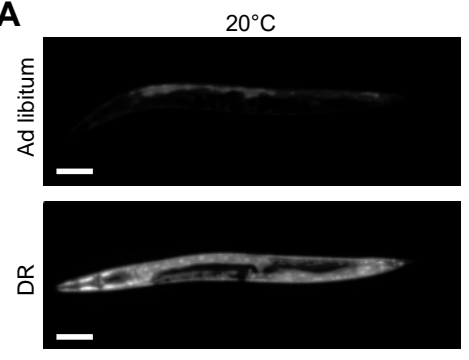

B
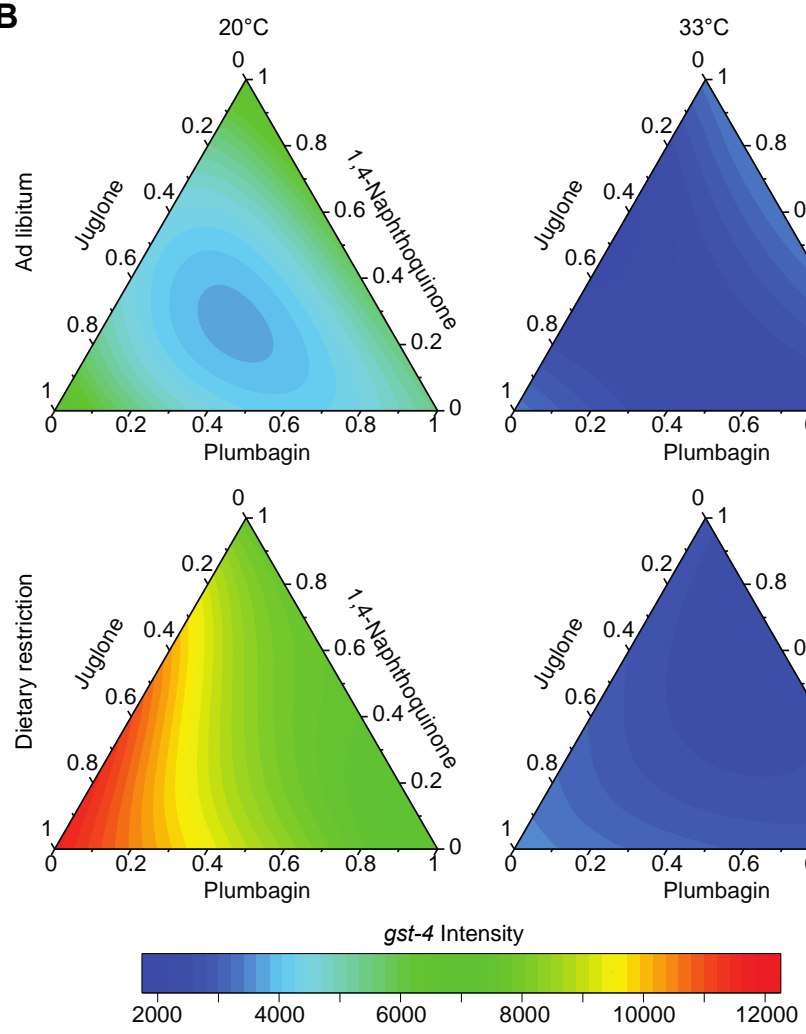

F

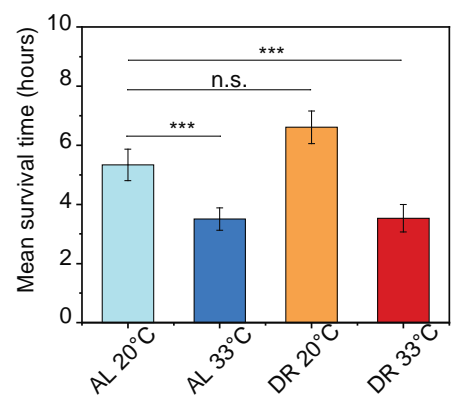

$33^{\circ} \mathrm{C}$
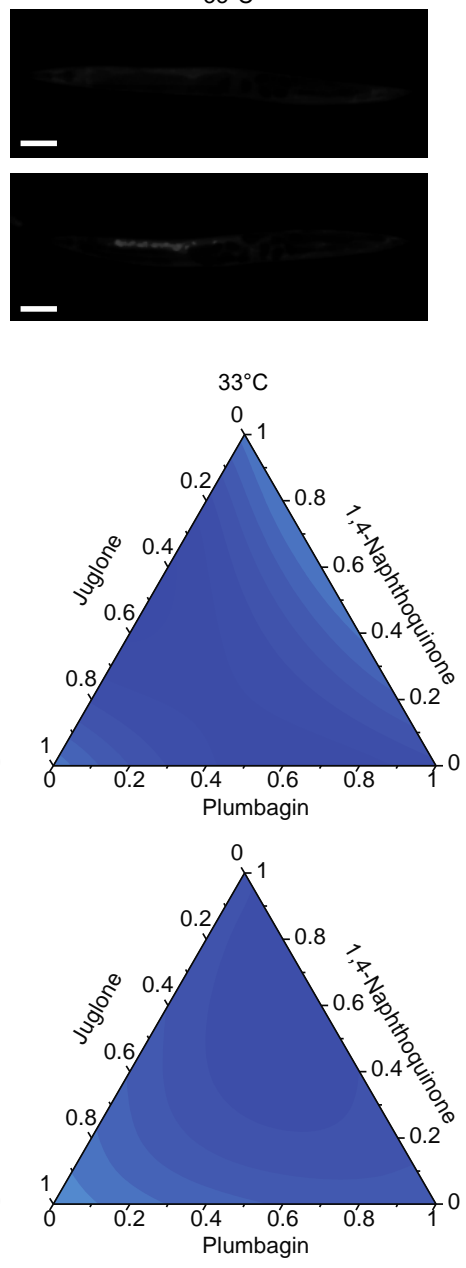

G

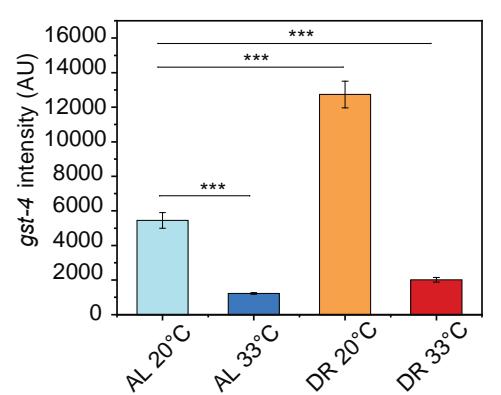

C

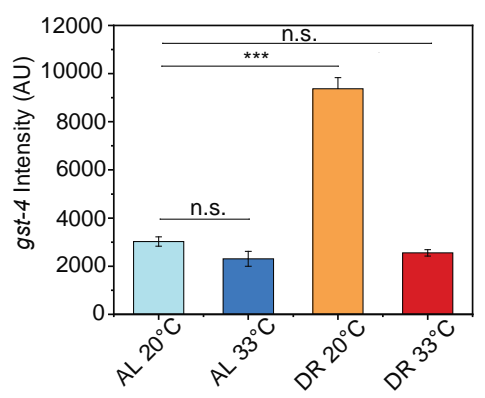

D

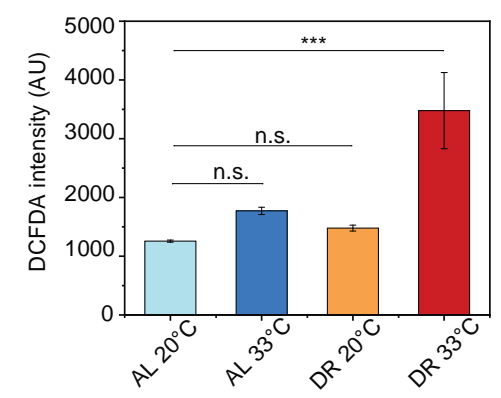

E

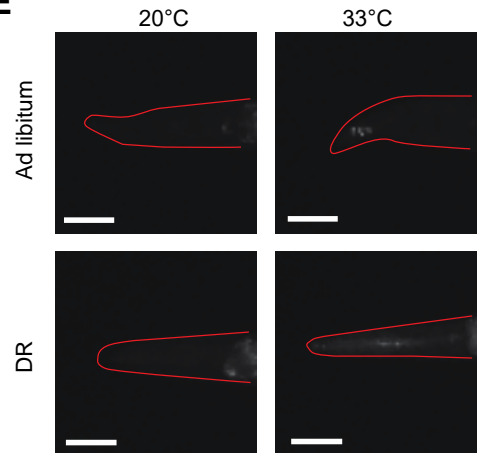

H

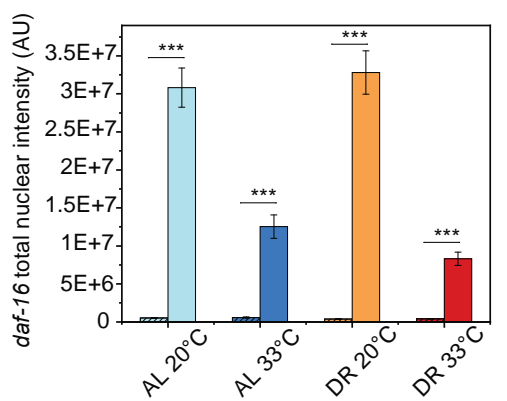

Figure 3: Naphthoquinone mixtures elicit differential response under simultaneous stress exposure.

A) Representative CL2166 animals exposed to ternary naphthoquinone mixtures (1,4-Naphthoquinone, Juglone, Plumbagin) at different environmental conditions. B) Response surface of $g s t-4$ expression levels for CL2166 animals under oxidative stress at different environmental conditions. C) $g s t-4$ expression levels for CL2166 animals under exposure to the ternary naphthoquinone mixture. D) DCFDA intensity levels for $\mathrm{N} 2$ animals exposed to the ternary naphthoquinone mixture. E) DCFDA staining of representative N2 
$\mu \mathrm{M}$ Juglone in CL2166 animals pre-exposed to the ternary naphthoquinone mixture at different environmental conditions. G) gst-4 expression levels under daf-16 RNAi for CL2166 animals exposed to ternary naphthoquinone mixtures. H) daf-16 expression levels for MAH97 animals exposed to ternary naphthoquinone mixtures, measured as total intensity in the nuclei of cells per worm. AL: ad libitum. DR: dietary restriction. $\mathrm{p}>0.05$ (n.s.), $\mathrm{p}<0.05(*), \mathrm{p}<0.001(* * *)$. p-values were calculated using Dunnett's test with ad libitum, $20^{\circ} \mathrm{C}$ as control after one-way ANOVA comparison in JMP 14.2.Response surfaces modeled using standard least squares second order Scheffe model where main effects and interactions were tested for significance (Table S2). Scale bars are $100 \mu \mathrm{m}$. Error bars are SEM. The experimentally acquired data is represented as conventional bar plots in Figure S5. 
A

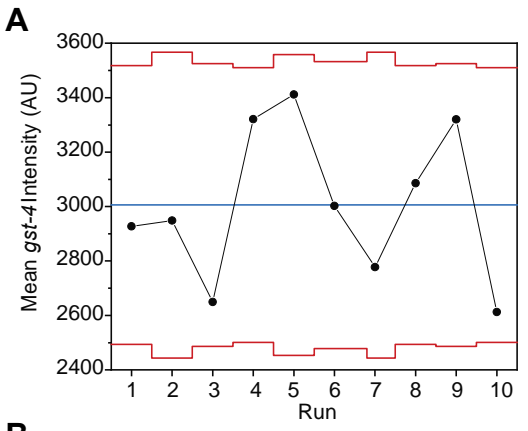

B
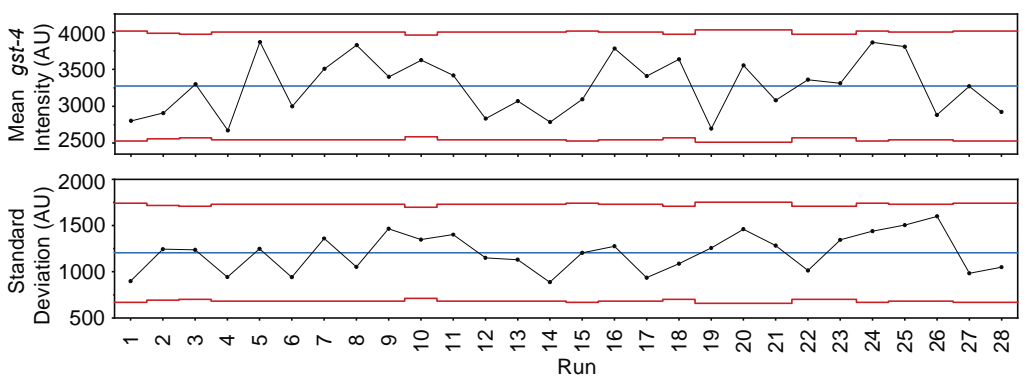

Figure S1: Xbar and S-charts for populations tested. A) Xbar and S-chart for 10 populations used for oxidative stress assays. B) Xbar and S-chart for 28 populations used for oxidative stress assays.

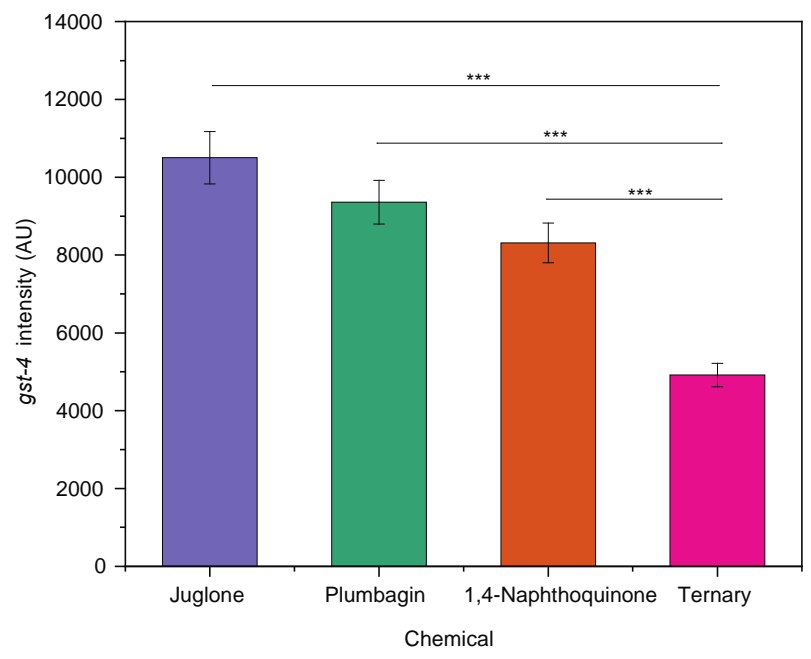

Figure S2: gst-4 response at $25^{\circ} \mathrm{C}$. gst-4 response to Plumbagin, 1, 4-Naphthoquinone, Juglone, and ternary mixture at $25^{\circ} \mathrm{C} . \mathrm{p}<0.001(* * *)$. Values follow the same trend as Figure $2 \mathrm{~B}$ with a lower value for the ternary mixture (middle point of response surface in Figure 2B), and higher values for individual naphthoquinones (vertex points of response surface in $2 \mathrm{~B}$ ). Error bars are SEM. 


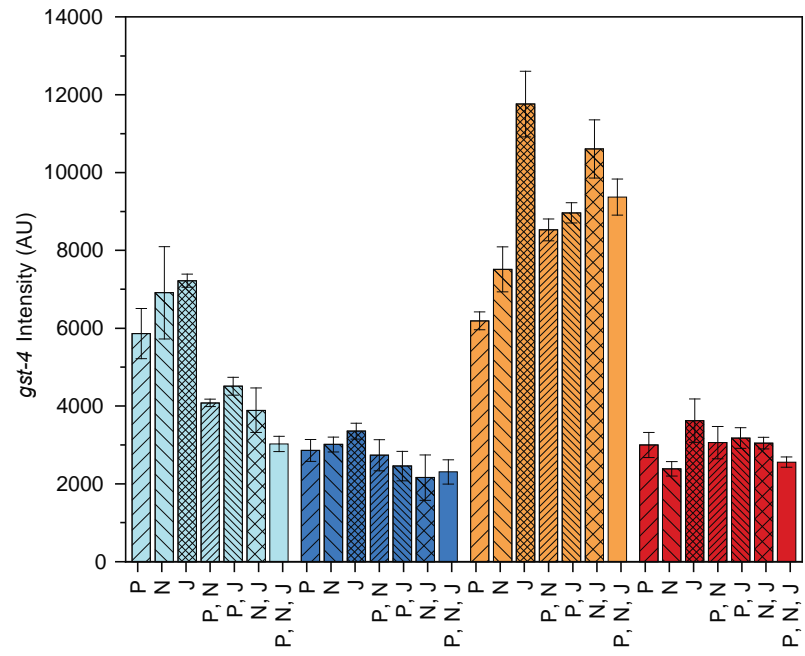

Mixture composition

Figure S3: Bar plot representation of experimentally acquired $g s t-4$ expression level. $g s t-4$ expression levels of animals exposed to naphthoquinone mixtures at different process conditions. From left to right, AL $20^{\circ} \mathrm{C}$ (light blue), AL $33^{\circ} \mathrm{C}$ (blue), DR $20^{\circ} \mathrm{C}$ (orange), DR $33^{\circ} \mathrm{C}$ (red). P-Plumbagin, N-1,4Naphthoquinone, J-Juglone. Error bars are SEM.
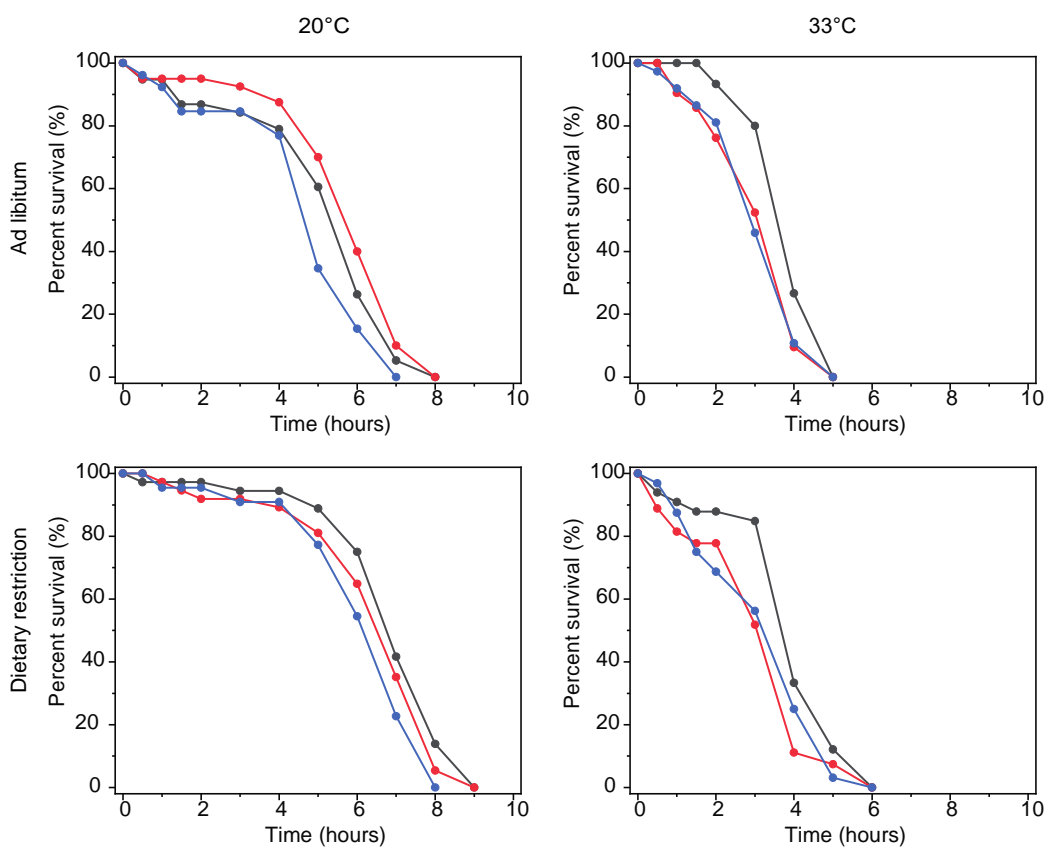

Figure S4: Lifespan curves for juglone survival assay. Lifespan curves built with OASIS 2 for CL2166 animals under ternary mixture at different environmental conditions. Each condition was tested three times. 
A

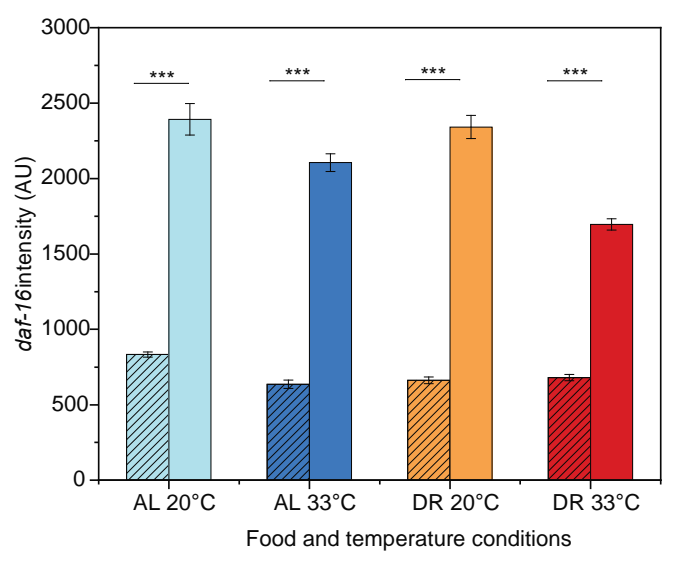

B

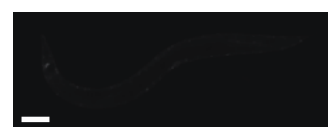

Figure S5: daf-16 response to ternary naphthoquinone mixture. A) daf-16 response to ternary mixture at different environmental conditions. Striated bars represent MAH97 animals under daf-16 RNAi, clear bars are control animals. B) Representative MAH97 animals under daf-16 RNAi (left) and control (right). $\mathrm{p}<0.001(* * *)$. Scale bars are $100 \mu \mathrm{m}$. Error bars are SEM.

\section{Acknowledgments}

Some strains were provided by the CGC, which is funded by NIH Office of Research Infrastructure Programs (P40 OD010440).

\section{Data availability}

All data underlying this work can be requested from the authors.

\section{Funding Statement}

This work was supported in part by the U.S. NIH grants R00AG046911 and R21AG059099; and by NCSU's Faculty Research and Professional Development program.

\section{Conflict of Interest Statement}

The authors declare no conflict of interest.

\section{Author contributions}

All authors designed the experiments and conceived the work. KSA and JH carried out experiments and analyzed data. JWS and KSA performed design of experiments and statistical analysis. KSA, JH, and ASM wrote the manuscript.

\section{References}

1. Chege PM, McColl G. Caenorhabditis elegans: A model to investigate oxidative stress and metal dyshomeostasis in Parkinson's disease. Front Aging Neurosci. 2014;6(MAY). doi:10.3389/fnagi.2014.00089

2. Chen X, Wang F, Hyun JY, et al. Recent progress in the development of fluorescent, luminescent and colorimetric probes for detection of reactive oxygen and nitrogen species. Chem Soc Rev. 2016;45(10):2976-3016. doi:10.1039/c6cs00192k 
3. Dias V, Junn E, Mouradian MM. The role of oxidative stress in parkinson's disease. J Parkinsons Dis. 2013;3(4):461-491. doi:10.3233/JPD-130230

4. Senchuk M, Dues D, Van Raamsdonk J. Measuring Oxidative Stress in Caenorhabditis elegans: Paraquat and Juglone Sensitivity Assays. Bio-Protocol. 2017;7(1). doi:10.21769/bioprotoc.2086

5. Altenburger R, Scholze M, Busch W, et al. Mixture effects in samples of multiple contaminants An inter-laboratory study with manifold bioassays. Environ Int. 2018;114:95-106. doi:10.1016/j.envint.2018.02.013

6. Goldberg MS. On the interpretation of epidemiological studies of ambient air pollution. In: Journal of Exposure Science and Environmental Epidemiology. Vol 17. Nature Publishing Group; 2007:S66-S70. doi:10.1038/sj.jes.7500629

7. Vermeulen R, Schymanski EL, Barabási AL, Miller GW. The exposome and health: Where chemistry meets biology. Science (80- ). 2020;367(6476):392-396. doi:10.1126/science.aay3164

8. Turner MC, Nieuwenhuijsen M, Anderson K, et al. Assessing the Exposome with External Measures: Commentary on the State of the Science and Research Recommendations. Annu Rev Public Health. 2017;38:215-239. doi:10.1146/annurev-publhealth-082516-012802

9. Grolig J, Wagner R. Naphthoquinones. In: Ullmann's Encyclopedia of Industrial Chemistry. Weinheim, Germany: Wiley-VCH Verlag GmbH \& Co. KGaA; 2000. doi:10.1002/14356007.a17_067

10. Shang Y, Chen C, Li Y, Zhao J, Zhu T. Hydroxyl radical generation mechanism during the redox cycling process of 1,4-naphthoquinone. Environ Sci Technol. 2012;46(5):2935-2942. doi:10.1021/es203032v

11. Finlayson-Pitts BJ, Pitts JN. Chemistry of the upper and lower atmosphere. Choice Rev Online. 2000;37(10):37-5725-37-5725. doi:10.5860/choice.37-5725

12. Preuss R, Koch HM, Wilhelm M, Pischetsrieder M, Angerer J. Pilot study on the naphthalene exposure of German adults and children by means of urinary 1- and 2-naphthol levels. Int J Hyg Environ Health. 2004;207(5):441-445. doi:10.1078/1438-4639-00313

13. Lin CY, Isbell MA, Morin D, et al. Characterization of a structurally intact in situ lung model and comparison of naphthalene protein adducts generated in this model vs lung microsomes. Chem Res Toxicol. 2005;18(5):802-813. doi:10.1021/tx049746r

14. Lin PH, Chen DR, Wang TW, Lin CH, Chuang MC. Investigation of the cumulative tissue doses of naphthoquinones in human serum using protein adducts as biomarker of exposure. Chem Biol Interact. 2009;181(1):107-114. doi:10.1016/j.cbi.2009.05.016

15. Wilson AS, Davis CD, Williams DP, Buckpitt AR, Pirmohamed M, Park BK. Characterisation of the toxic metabolite(s) of naphthalene. Toxicology. 1996;114(3):233-242. doi:10.1016/S0300$483 X(96) 03515-9$

16. Inbaraj JJ, Chignell CF. Cytotoxic Action of Juglone and Plumbagin: A Mechanistic Study Using HaCaT Keratinocytes. Chem Res Toxicol. 2004;17(1):55-62. doi:10.1021/tx034132s

17. Fowler P, Meurer K, Honarvar N, Kirkland D. A review of the genotoxic potential of 1,4naphthoquinone. Mutat Res - Genet Toxicol Environ Mutagen. 2018;834:6-17. doi:10.1016/j.mrgentox.2018.07.004

18. Hunt PR, Son TG, Wilson MA, et al. Extension of lifespan in C. elegans by naphthoquinones that 
act through stress hormesis mechanisms. PLoS One. 2011;6(7):21922. doi:10.1371/journal.pone.0021922

19. Kumagai Y, Shinkai Y, Miura T, Cho AK. The chemical biology of naphthoquinones and its environmental implications. Annu Rev Pharmacol Toxicol. 2012;52:221-247. doi:10.1146/annurev-pharmtox-010611-134517

20. Esnault S, Rosenthal LA, Shen ZJ, et al. A critical role for Pin1 in allergic pulmonary eosinophilia in rats. J Allergy Clin Immunol. 2007;120(5):1082-1088. doi:10.1016/j.jaci.2007.06.024

21. Corsi AK, Wightman B, Chalfie M. A transparent window into biology: A primer on Caenorhabditis elegans. Genetics. 2015;200(2):387-407. doi:10.1534/genetics.115.176099

22. Wittkowski P, Marx-Stoelting P, Violet N, et al. Caenorhabditis elegans As a Promising Alternative Model for Environmental Chemical Mixture Effect Assessment - A Comparative Study. Environ Sci Technol. 2019;53(21):12725-12733. doi:10.1021/acs.est.9b03266

23. Vingskes AK, Spann N. The toxicity of a mixture of two antiseptics, triclosan and triclocarban, on reproduction and growth of the nematode Caenorhabditis elegans. Ecotoxicology. 2018;27(4):420429. doi:10.1007/s10646-018-1905-9

24. Lu C, Svoboda KR, Lenz KA, Pattison C, Ma H. Toxicity interactions between manganese (Mn) and lead $(\mathrm{Pb})$ or cadmium $(\mathrm{Cd})$ in a model organism the nematode C. elegans. Environ Sci Pollut Res. 2018;25(16):15378-15389. doi:10.1007/s11356-018-1752-5

25. Chen $\mathrm{H}$, Wang $\mathrm{C}, \mathrm{Li} \mathrm{H}$, et al. A review of toxicity induced by persistent organic pollutants (POPs) and endocrine-disrupting chemicals (EDCs) in the nematode Caenorhabditis elegans. $J$ Environ Manage. 2019;237:519-525. doi:10.1016/j.jenvman.2019.02.102

26. Hunt PR. The C. elegans model in toxicity testing. J Appl Toxicol. 2017;37(1):50-59. doi: $10.1002 /$ jat.3357

27. Boyd WA, Smith M V., Co CA, et al. Developmental effects of the ToxCast ${ }^{\mathrm{TM}}$ phase I and phase II chemicals in caenorhabditis elegans and corresponding responses in Zebrafish, Rats, and Rabbits. Environ Health Perspect. 2016;124(5):586-593. doi:10.1289/ehp.1409645

28. Allard P, Kleinstreuer NC, Knudsen TB, Colaiácovo MP. A C. elegans screening platform for the rapid assessment of chemical disruption of germline function. Environ Health Perspect. 2013;121(6):717-724. doi:10.1289/ehp.1206301

29. Klaus V, Hartmann T, Gambini J, et al. 1,4-Naphthoquinones as inducers of oxidative damage and stress signaling in HaCaT human keratinocytes. Arch Biochem Biophys. 2010;496(2):93-100. doi:10.1016/j.abb.2010.02.002

30. Blackwell TK, Steinbaugh MJ, Hourihan JM, Ewald CY, Isik M. SKN-1/Nrf, stress responses, and aging in Caenorhabditis elegans. Free Radic Biol Med. 2015;88(Part B):290-301. doi:10.1016/j.freeradbiomed.2015.06.008

31. Wu M, Kang X, Wang Q, Zhou C, Mohan C, Peng A. Regulator of G protein signaling-1 modulates paraquat-induced oxidative stress and longevity via the insulin like signaling pathway in Caenorhabditis elegans. Toxicol Lett. 2017;273:97-105. doi:10.1016/j.toxlet.2017.03.027

32. Inoue H, Hisamoto N, Jae HA, et al. The C. elegans p38 MAPK pathway regulates nuclear localization of the transcription factor SKN-1 in oxidative stress response. Genes Dev. 2005;19(19):2278-2283. doi:10.1101/gad.1324805 
33. Przybysz AJ, Choe KP, Roberts LJ, Strange K. Increased age reduces DAF-16 and SKN-1 signaling and the hormetic response of Caenorhabditis elegans to the xenobiotic juglone. Mech Ageing Dev. 2009;130(6):357-369. doi:10.1016/j.mad.2009.02.004

34. Benedetto A, Au C, Avila DS, Milatovic D, Aschner M. Extracellular dopamine potentiates Mninduced oxidative stress, lifespan reduction, and dopaminergic neurodegeneration in a BLI-3dependent manner in caenorhabditis elegans. Ashrafi K, ed. PLoS Genet. 2010;6(8):e1001084. doi:10.1371/journal.pgen.1001084

35. Possik E, Pause A. Measuring Oxidative Stress Resistance of Caenorhabditis elegans in 96-well Microtiter Plates. J Vis Exp. 2015;99(99):527463791-52746. doi:10.3791/52746

36. Hasegawa K, Miwa S, Isomura K, Tsutsumiuchi K, Taniguchi H, Miwa J. Acrylamide-responsive genes in the nematode Caenorhabditis elegans. Toxicol Sci. 2008;101(2):215-225. doi:10.1093/toxsci/kfm276

37. Crombie TA, Tang L, Choe KP, Julian D. Inhibition of the oxidative stress response by heat stress in Caenorhabditis elegans. J Exp Biol. 2016;219(14):2201-2211. doi:10.1242/jeb.135327

38. Tawe WN, Eschbach ML, Walter RD, Henkle-Dührsen K. Identification of stress-responsive genes in Caenorhabditis elegans using RT-PCR differential display. Nucleic Acids Res. 1998;26(7):1621-1627. doi:10.1093/nar/26.7.1621

39. Link CD, Johnson CJ. Reporter transgenes for study of oxidant stress in Caenorhabditis elegans. In: Methods in Enzymology. Vol 353. Academic Press Inc.; 2002:497-505. doi:10.1016/S00766879(02)53072-X

40. Leiers B, Kampkötter A, Grevelding CG, Link CD, Johnson TE, Henkle-Dührsen K. A stressresponsive glutathione $S$-transferase confers resistance to oxidative stress in Caenorhabditis elegans. Free Radic Biol Med. 2003;34(11):1405-1415. doi:10.1016/S0891-5849(03)00102-3

41. Goos P, Jones B. Optimal Design of Experiments: A Case Study Approach.; 2011. doi:10.1002/9781119974017

42. Goos P, Donev AN. The D-optimal design of blocked experiments with mixture components. $J$ Qual Technol. 2006;38(4):319-332. doi:10.1080/00224065.2006.11918621

43. Porta-de-la-Riva M, Fontrodona L, Villanueva A, Cerón J. Basic Caenorhabditis elegans methods: Synchronization and observation. J Vis Exp. 2012;(64). doi:10.3791/4019

44. Stiernagle T. Maintenance of C. elegans. WormBook. 2006. doi:10.1895/wormbook.1.101.1

45. Keith SA, Amrit FRG, Ratnappan R, Ghazi A. The C. elegans healthspan and stress-resistance assay toolkit. Methods. 2014;68(3):476-486. doi:10.1016/j.ymeth.2014.04.003

46. Fraser AG, Kamath RS, Zipperlen P, Martinez-Campos M, Sohrmann M, Ahringer J. Functional genomic analysis of C. elegans chromosome I by systematic RNA interference. Nature. 2000;408(6810):325-330. doi:10.1038/35042517

47. Kamath RS, Fraser AG, Dong Y, et al. Systematic functional analysis of the Caenorhabditis elegans genome using RNAi. Nature. 2003;421(6920):231-237. doi:10.1038/nature01278

48. Yoon D, Lee M-H, Cha D. Measurement of Intracellular ROS in Caenorhabditis elegans Using 2',7'-Dichlorodihydrofluorescein Diacetate. Bio-Protocol. 2018;8(6). doi:10.21769/bioprotoc. 2774

49. Lu T, Aron L, Zullo J, et al. REST and stress resistance in ageing and Alzheimer's disease. 
50. Han SK, Lee D, Lee H, et al. OASIS 2: Online application for survival analysis 2 with features for the analysis of maximal lifespan and healthspan in aging research. Oncotarget. 2016;7(35):5614756152. doi:10.18632/oncotarget.11269

51. Preece DA, Cornell JA. Experiments with Mixtures: Designs, Models, and the Analysis of Mixture Data. Biometrics. 1982;38(1):288. doi:10.2307/2530325

52. Scouse RA. Introduction to Statistical Quality Control. Plast rubber Int. 1985;10(1):30-32. doi: $10.2307 / 2988304$

53. Hasegawa K, Miwa S, Tajima T, Tsutsumiuchi K, Taniguchi H, Miwa J. A rapid and inexpensive method to screen for common foods that reduce the action of acrylamide, a harmful substance in food. Toxicol Lett. 2007;175(1-3):82-88. doi:10.1016/j.toxlet.2007.09.013

54. Van Raamsdonk JM, Hekimi S. Superoxide dismutase is dispensable for normal animal lifespan. Proc Natl Acad Sci. 2012;109(15):5785-5790. doi:10.1073/pnas.1116158109

55. Paek J, Lo JY, Narasimhan SD, et al. Mitochondrial SKN-1/Nrf mediates a conserved starvation response. Cell Metab. 2012;16(4):526-537. doi:10.1016/j.cmet.2012.09.007

56. Rollins JA, Shaffer D, Snow SS, Kapahi P, Rogers AN. Dietary restriction induces posttranscriptional regulation of longevity genes. Life Sci Alliance. 2019;2(4). doi:10.26508/lsa.201800281

57. Schulz TJ, Zarse K, Voigt A, Urban N, Birringer M, Ristow M. Glucose Restriction Extends Caenorhabditis elegans Life Span by Inducing Mitochondrial Respiration and Increasing Oxidative Stress. Cell Metab. 2007;6(4):280-293. doi:10.1016/j.cmet.2007.08.011

58. Gusarov I, Pani B, Gautier L, et al. Glycogen controls Caenorhabditis elegans lifespan and resistance to oxidative stress. Nat Commun. 2017;8(1):1-12. doi:10.1038/ncomms15868

59. Greer EL, Brunet A. Different dietary restriction regimens extend lifespan by both independent and overlapping genetic pathways in C. elegans. Aging Cell. 2009;8(2):113-127. doi:10.1111/j.1474-9726.2009.00459.x

60. Lee RYN, Hench J, Ruvkun G. Regulation of C. elegans DAF-16 and its human ortholog FKHRL1 by the daf-2 insulin-like signaling pathway. Curr Biol. 2001;11(24):1950-1957. doi:10.1016/S0960-9822(01)00595-4

61. Lin K, Hsin H, Libina N, Kenyon C. Regulation of the Caenorhabditis elegans longevity protein DAF-16 by insulin/IGF-1 and germline signaling. Nat Genet. 2001;28(2):139-145. doi: $10.1038 / 88850$

62. Bishop NA, Guarente L. Two neurons mediate diet-restriction-induced longevity in C. elegans. Nature. 2007;447(7144):545-549. doi:10.1038/nature05904

63. Hernández-Muñoz LS, Gómez M, González FJ, González I, Frontana C. Towards a molecularlevel understanding of the reactivity differences for radical anions of juglone and plumbagin: An electrochemical and spectroelectrochemical approach. Org Biomol Chem. 2009;7(9):1896-1903. doi:10.1039/b822684a

64. O'Brien PJ. Molecular mechanisms of quinone cytotoxicity. Chem Biol Interact. 1991;80(1):1-41. doi:10.1016/0009-2797(91)90029-7

65. Seshadri P, Rajaram A, Rajaram R. Plumbagin and juglone induce caspase-3-dependent apoptosis 
involving the mitochondria through ROS generation in human peripheral blood lymphocytes. Free Radic Biol Med. 2011;51(11):2090-2107. doi:10.1016/j.freeradbiomed.2011.09.009

630

631

632

633

634

635

636

637

638
66. Benedetto A, Bambade T, Au C, et al. New label-free automated survival assays reveal unexpected stress resistance patterns during C. elegans aging. Aging Cell. 2019;18(5). doi:10.1111/acel.12998

67. Tullet JMA. DAF-16 target identification in C. elegans: past, present and future. Biogerontology. 2015;16(2):221-234. doi:10.1007/s10522-014-9527-y

68. Bhatla N, Horvitz HR. Light and Hydrogen Peroxide Inhibit C.elegans Feeding through Gustatory Receptor Orthologs and Pharyngeal Neurons. Neuron. 2015;85(4):804-818. doi:10.1016/j.neuron.2014.12.061 